\title{
Transmission Power Selection for Ad hoc Networks
}

\author{
Jianping He, Jiahai Yang*, Changqing An, Xuenong Li \\ Network Research Center, Tsinghua University \\ Tsinghua National Laboratory for Information Science and Technology, \\ Beijing, P.R. China, 100084 \\ hejp06@mails.tsinghua.edu.cn, yang@cernet.edu.cn, \{acq, Ixn\}@tsinghua.edu.cn
}

\begin{abstract}
In wireless ad hoc networks, as an important technology, power control is widely used to increase energy efficiency and improve network throughput. Power control is composed of two components: one component handles the problems caused by asymmetric channel; the other component is responsible for selecting transmission power. In this paper, we focus on transmission power selection for ad hoc networks using 802.11 DCF MAC protocol.

In this paper, we model the network with power control in joint with PHY layer and MAC layer. Then we prove that in considering the noise, interference from other nodes and packet length, it is NP-hard to obtain the optimal transmission power for every node even if it knows the complete knowledge of the network. After that, we propose two heuristic transmission power selection algorithms, one for energy-oriented power control and the other for throughputoriented power control, which are time efficiency and able to improve the network performance largely. Finally, we evaluate our algorithms under different topologies and in different packet size. The results show that our algorithms can improve the network performance largely when compared with 802.11 and traditional power control algorithms.
\end{abstract}

\section{Keywords}

Ad hoc networks, BER, SINR, Power selection

\section{INTRODUCTION}

In recent years, wireless ad hoc networks have gained tremendous attention due to their wide applications in civilian and military and their capability of building networks without the need for a pre-existing infrastructure. In ad hoc networks, energy efficiency and throughput are two most important metrics to evaluate its performance. To better understand how the energy serves the MAC layer, energy efficiency is evaluated by the number of valid bits transmitted successfully per Joule energy consumed, and throughput is evaluated by the number of valid bits transmitted successfully by the network in a period of time, say 1s. In this

Permission to make digital or hard copies of all or part of this work for personal or classroom use is granted without fee provided that copies are not made or distributed for profit or commercial advantage and that copies bear this notice and the full citation on the first page. To copy otherwise, to republish, to post on servers or to redistribute to lists, requires prior specific permission and/or a fee.

WICON'08, November 17-19, 2008, Maui, Hawaii, USA

Copyright 2008 ICST 978-963-9799-36-3 ...\$5.00. paper, we say valid bits means that the payload bit of MAC packet, which is similar to literature [1]. In Wireless Sensor Networks (WSNs), the most important performance metric is energy efficiency. While for some applications, such as File Transport Protocol(FTP), voice and video applications, throughput is more important than energy consumption.

\subsection{Motivation}

Power control is a technology that attempts to conserve energy consumption and improve network throughput. In MAC layer, power control can be mainly classified into energyoriented and throughput-oriented according to its application context. The energy-oriented power control takes energy reduction as its main goal. While throughput-oriented power control takes throughput increasing as its main goal. Generally, power control is composed of two components no matter what the objective is. One component deals with the problems caused by asymmetric channel and the other component is responsible for selecting transmission power. In this paper, we focus on the latter component.

Transmission power selection is very important to improve the performance of power control. Because of the intrinsic nature of wireless channel, such as broadcast and high BitError-Rate (BER), power control may reduce the Signal-toInterference-and-Noise-Ratio (SINR) at the receiver largely. A lower SINR results in a higher BER which will cause more packet retransmission. And retransmission has a counter effect to the network performance. Thus, selecting an inappropriate transmission power may worsen the network performance instead of improving it. For more detail, we take a two-node topology as an example to show how an inappropriate transmission power worsens the network performance.

We use a set of typical numeric values. The distance between $\mathrm{S}$ and $\mathrm{D}$ is $60 \mathrm{~m}$ and current noise is $1.0 \mathrm{e}-10 \mathrm{~W}$ ($70 \mathrm{dBm})$. The other parameters are discussed in Section 4.2. Node $\mathrm{S}$ transmits using the minimum power which ensures the packet will be received by node D in PHY layer, i.e., the signal power at node $\mathrm{D}$ equals to the receiving threshold power. Therefore, SINR at node D is 4.46 and BER is 0.0014 . Hence, a packet with 500 bytes (4000 bits) should retransmit more than 270 times in average ${ }^{1}$. While using standard 802.11 protocol, the packet is just retransmitted approximately once in average. Therefore, this power control protocol consumes 15 times energy in comparison with 802.11 protocol.

The scenario discussed above is the easiest one because there is no interference from other nodes in this topology. With the increase of node number, the problem of select-

\footnotetext{
${ }^{1}$ Here, we suppose the packet will be retransmitted until it is received successfully.
} 
ing an appropriate transmission power becomes much more complex. In this paper, we aim to solve this problem. Specifically, our contributions are:

- We propose power control models for energy and throughput oriented power control. Taking into account of the channel noise and interference from other nodes, we model the power control algorithms for wireless ad hoc network and express the specific metric (energy efficiency or throughput) as a function of noise, transmission power, coordinates of other node and the packet size to be transmitted, under the assumption that the channel is an Additive White Gaussian Noise channel (AWGN) and the transceiver uses Binary Phase Shift Keying (BPSK) modulator ${ }^{2}$.

- Based on the analysis, we prove that obtaining the optimal transmission power for energy-oriented power control and throughput-oriented power control are NPhard even if every node knows the complete knowledge of the network.

- We propose heuristic algorithms to obtain the transmission power that are local, time efficient and performance improvement, for the two kinds of power control, respectively. By simulating in different topologies and different packet sizes, our algorithms yield good performance than the power selection algorithms ever before.

\subsection{Background}

In this subsection, we will review some background knowledge to help us analyze the power control scheme and understand the PHY layer.

In wireless environment, the receiving power decays with the increase of transmission distance. In this paper, the most widely used model in engineering, a simplified distanceloss model, is used to describe the wireless channel. In this model, the receiving power at distance $d$ is [2]

$$
p_{r}=p_{t} \frac{\lambda^{2} d_{0}^{\delta-2}}{16 \pi^{2} d^{\delta}}
$$

where $p_{t}$ is the transmission power; $p_{r}$ is the receiving power; $\lambda$ is the wavelength of the electromagnetic wave; $d_{0}$ is the reference distance of the antenna; and $\delta$ is the pass-loss exponent. $\delta$ is a constant relative to the environment, and ranges from 2 to $6 . d_{0}$ ranges from $1 \mathrm{~m}$ to $10 \mathrm{~m}$ for indoor environment and $10 \mathrm{~m}$ to $100 \mathrm{~m}$ for outdoor. In this paper, we let $\delta=2$, and $d_{0}=1 \mathrm{~m}$.

BER is an important parameter in wireless communication. The research reveals that BER is a function of SINR [3]. The expression is different for different modulation algorithms. In our analysis, we use Binary Phase Shift Keying (BPSK) because it is a basic modulation algorithm and used by almost all the wireless network cards. In AGWN channel with BPSK modulator, the research in [3] reveals that

$$
B E R=Q(\sqrt{2 \gamma})
$$

where $\gamma$ is $\operatorname{SINR}$. Function $Q$ is defined as

$$
Q(x)=\int_{x}^{+\infty} \frac{1}{\sqrt{2 \pi}} \exp \left(\frac{-x^{2}}{2}\right) d x .
$$

\footnotetext{
${ }^{2}$ This paper can be easily extend to other modulation algorithms.
}

The remainder of this paper is organized as follows. In section 2, we model the power control algorithms for wireless ad hoc networks. In this section, we prove that obtaining the optimal transmission power is NP-hard for energy-oriented and throughput-oriented power control scheme, even if every node knows the complete knowledge of the network. Following that, two heuristic distributed algorithms are proposed for energy-oriented and throughput-oriented power control in section 3. In section 4, our proposed algorithms are evaluated by simulations. The related works are summarized in section 5. Finally, we conclude the paper in section 6 .

\section{POWER CONTROL MODEL}

In this section, we study how the transmission power, noise and node interference, node distance and packet size impact the performance of power control.

\subsection{Network Model}

The network is a bidirectional graph $\mathcal{G}=(\mathcal{V}, \mathcal{E})$, where $\mathcal{V}$ and $\mathcal{E}$ represent the set of nodes and edges respectively, i.e., $\mathcal{V}=\left\{v_{1}, v_{2}, v_{3}, \ldots, v_{n}\right\}$ and $\mathcal{E}=\left\{e_{1}, e_{2}, e_{3}, \ldots, e_{k}\right\}$. The coordinates of node $v_{i}$ and $v_{j}$ are $C_{i}=\left(x_{i}, y_{i}\right), C_{j}=\left(x_{j}, y_{j}\right)$.

$$
e_{m}=\left(v_{i}, v_{j}\right), \text { iff } \frac{p_{\max } \lambda^{2}}{16 \pi^{2}\left|C_{i}-C_{j}\right|^{2}} \geq p_{r t h},
$$

where $p_{\max }$ and $p_{\text {rth }}$ are the maximum transmission power and receiving threshold respectively. $\left|C_{i}-C_{j}\right|$ is the Euler distance of $v_{i}$ and $v_{j}$. Eq.(4) means that $v_{i}$ and $v_{j}$ are in the transmission range of each other when using the maximum transmission power ${ }^{3}$.

In the network, a node $v_{i}$ is featured by the following parameters. $p_{t x}^{i}$ is the transmission power; $S_{p k t}^{i}$ is the length of DATA packet; $C_{i}$ is the Coordinate, where $C_{i}=\left(x_{i}, y_{i}\right)$; $S_{o h}$ is the length of packet overhead, which is a constant and its length is defined by MAC protocol.

In this paper, we classify the node interferences into active interference and potential interference. Active interference is the one from the active nodes and potential interference is the one from the potential nodes which are defined as the nodes going to access the channel. Active nodes compose the active set $\mathcal{A}$ and potential nodes compose potential set $\mathcal{P}$.

\subsection{Energy-oriented power control model}

In energy-oriented power control, the main purpose is to minimize energy consumption for transmitting a valid bit successfully. Hence, in this subsection, the metric to evaluate the performance of power control is the energy consumed for transmitting a valid bit successfully.

We suppose there are $k_{1}$ active nodes and $k_{2}$ potential nodes during the interval that the receiver receives the packet, i.e., $\mathcal{A}=\left\{v_{1}, v_{2}, \ldots, v_{k_{1}}\right\}, \mathcal{P}=\left\{v_{k_{1}+1}, v_{k_{2}}, \ldots, v_{k_{1}+k_{2}}\right\}$. In the next, we suppose that node $v_{i}$ sends a packet to node $v_{j}$. The noise and interference power $p_{I N}^{j}\left(P_{t x}\right)$ at $v_{j}$ is

$$
\begin{aligned}
p_{I N}^{j}\left(P_{t x}\right)= & p_{\text {noise }}+\sum_{\forall v_{l} \in \mathcal{A} \backslash v_{j}} \frac{p_{t_{l}} \lambda^{2}}{16 \pi^{2}\left|C_{j}-C_{l}\right|^{2}} \\
& +\sum_{\forall v_{l} \in \mathcal{P} \backslash v_{j}} \frac{p_{t_{l}} \lambda^{2}}{16 \pi^{2}\left|C_{j}-C_{l}\right|^{2}},
\end{aligned}
$$

where $p_{\text {noise }}$ is the power of Gaussian white noise, $P_{t x}=$ $\left(p_{t x}^{1}, p_{t x}^{2}, \ldots, p_{t x}^{k_{1}}, p_{t x}^{k_{1}+1}, \ldots, p_{t x}^{k_{1}+k_{2}}\right)$ is the transmission power ${ }^{3}$ MAC layer power control does not influence the network
connectivity 
vector for $v_{1}, v_{2}, \ldots, v_{k_{1}+k_{2}}$. In Eq.(5), the second term is the interference from active set and the third term is the one from potential set, respectively. In practice, 802.11 [4] uses CSMA/CA scheme, and samples the channel periodically to detect if the channel is idle. Hence, the first two terms can be sampled by the network card. Thus, Eq.(5) can be rewritten as:

$$
p_{I N}^{j}\left(P_{t x}\right)=p_{s}+\sum_{\forall v_{l} \in \mathcal{P} \backslash v_{j}} \frac{p_{t_{l}} \lambda^{2}}{16 \pi^{2}\left|C_{j}-C_{l}\right|^{2}},
$$

where $p_{s}$ is the power sampled by network card. Therefore, SINR at node $v_{j}$ is

$$
\gamma\left(P_{t x}\right)=\frac{p_{r}\left(p_{t}^{i}\right)}{p_{I N}^{j}\left(P_{t x}\right)} .
$$

Then, BER at node $v_{j}$ is

$$
B E R\left(P_{t x}\right)=Q\left(\sqrt{2 \gamma\left(P_{t x}\right)}\right) .
$$

The probability that the packet from $v_{i}$ received by $v_{j}$ is

$$
p_{p k t}^{i}\left(P_{t x}\right)=\left(1-B E R\left(P_{t x}\right)\right)^{S_{p k t}^{i}} .
$$

Therefore, packet $S_{p k t}^{i}$ should be retransmitted $n_{i}\left(P_{t x}\right)$ times until received successfully, where

$$
n_{i}\left(P_{t x}\right)=\sum_{k=1}^{+\infty} p_{p k t}^{i}\left(P_{t x}\right)\left(1-p_{p k t}^{i}\left(P_{t x}\right)\right)^{k-1}=\frac{1}{p_{p k t}^{i}\left(P_{t x}\right)}
$$

When $S_{p k t}^{1}, S_{p k t}^{2}, \ldots, S_{p k t}^{k_{1}+k_{2}}$ are received successfully, the energy consumption is

$$
E\left(P_{t x}\right)=\sum_{i=1}^{k_{1}+k_{2}} n_{i}\left(P_{t x}\right) \frac{S_{p k t}^{i} p_{t x}^{i}}{B}
$$

where $B$ is the bandwidth. Therefore, the energy consumed for transmitting a valid bit successfully is

$$
e\left(P_{t x}\right)=\frac{E\left(P_{t x}\right)}{\sum_{i=1}^{k_{1}+k_{2}}\left(S_{p k t}^{i}-S_{o h}\right)} .
$$

As any two node $v_{i i}$ and $v_{j j}$ that can transmit simultaneously should not be in the transmission range of each other, we have, $\forall v_{i i}, v_{j j} \in A \cup P$

$$
\begin{aligned}
& p_{t x}^{i i} \frac{\lambda^{2}}{16 \pi^{2}\left|C_{i i}-C_{j j}\right|^{2}}<p_{r t h} \\
& p_{t x}^{j j} \frac{\lambda^{2}}{16 \pi^{2}\left|C_{i i}-C_{j j}\right|^{2}}<p_{r t h},
\end{aligned}
$$

In current practice, there are only a fixed number of power levels available. The $i$ th power level is denoted as $p_{l}^{i}$. To simplify our analysis, we suppose that there is a 0 power level, i.e., $p_{l}^{0}=0$, in case that a node sets its transmission power as 0 . In this paper, we suppose the interval between two adjacent power levels is the same except power level 0 . Thus, $\forall i, j \in(1,2, \ldots, n), p_{l}^{i+1}-p_{l}^{i}=p_{l}^{j+1}-p_{l}^{j}$, where $\mathrm{n}$ is the number of power level.

\subsection{Throughput-oriented power control model}

Power control not only improve energy efficiency but also increase network capacity [5] which contributes to network throughput. In this subsection, we define the valid network throughput as follows:

$$
T=\sum_{i=1}^{k_{1}+k_{2}} t_{i}\left(P_{t x}\right)
$$

where $t_{i}$ is the channel throughput of $\left(v_{i}, v_{j}\right)$. Based on the analysis in subsection 2.2 , we have

$$
t_{i}\left(P_{t x}\right)=\frac{S_{p k t}^{i}-S_{o h}}{S_{p k t}^{i}} \times \frac{B}{n_{i}\left(P_{t x}\right)},
$$

where $\frac{S_{p k t}^{i}-S_{o h}}{S_{p k t}^{i}}$ is the packet valid data efficiency, i.e., the efficiency of transmitting a packet if it is received successfully.

\subsection{Complexity of power selection}

Based on the former power control models, we get the following theorems.

THEOREM 1. It is NP-hard to obtain the optimal transmission power for energy-oriented power control even if every node knows the complete knowledge of the network.

THEOREM 2. Obtaining the optimal transmission power in throughput-oriented is NP-hard though every node knows the complete knowledge of the network.

Due to the space limitation of this paper, we skip the proof of these theorems in this paper. For more details, the reader can refer [6]. So, obtaining the optimal transmission power is very difficult mainly for two reasons: 1) it is hard for a node to know the complete knowledge of the network; 2) on the premise that every node knows the complete knowledge of the network, there is no efficient algorithm to compute the optimal power. Therefore, in the next section, we will propose two power selection algorithms, one is Power Selection algorithm for Energy-oriented power control (PSE) and the other is Power Selection algorithm for Throughput-oriented power contorl (PST).

\section{HEURISTIC TRANSMISSION POWER SE- LECTION ALGORITHM}

In section 2, we know that it is difficult to obtain the optimal transmission power in power control. In this section, by ignoring the potential interference, we propose two $O(1)$ heuristic algorithms for energy-oriented and throughput oriented power control, respectively. The potential interference is ignored because:

- It is hard to know the transmission power of the potential node set $\mathcal{P}$. We suppose that, fortunately, there is a central node which communicates with all the other nodes directly ${ }^{4}$. It needs $2(n-1)$ time slots for the node to know the transmission power of other nodes under the assumption that every node has selected a transmission power, where $n$ is the size of potential set.

Thus, Eq.(6) can be rewritten as

$$
p_{I N}^{j}=p_{s}
$$

\subsection{Transmission power for energy-oriented power selection}

In this subsection, we will propose an $O(1)$ distributed algorithm, Power Selection algorithm for Energy-oriented

\footnotetext{
${ }^{4}$ This central node is similar to the base station in Cellular Network. But for ad hoc networks, such central node is unpractical.
} 
power control (PSE), to obtain the transmission power. Every node makes decision locally because it is difficult for a node to know the complete knowledge of the network. Hence, we propose an algorithm that every node sets its transmission power to maximize its own energy efficiency.

For a node, the average energy consumed for transmitting a packet is

$$
E\left(p_{t x}\right)=n_{i}\left(p_{t x}\right) \frac{S_{p k t} p_{t x}}{B}=\frac{S_{p k t} p_{t x}}{B\left(1-Q\left(\sqrt{2 \gamma\left(p_{t x}\right)}\right)\right)^{S_{p k t}}}
$$

In Eq.(17) and the following equations, the superscript is omitted. According to Eq.(17), we have

$$
e\left(p_{t x}\right)=\frac{S_{p k t} p_{t x}}{B\left(S_{p k t}-S_{o h}\right)\left(1-Q\left(\sqrt{2 \gamma\left(p_{t x}\right)}\right)\right)^{S_{p k t}}} .
$$

The differential coefficient of Eq.(18) is

$$
\begin{aligned}
\frac{d e\left(p_{t x}\right)}{d p_{t x}}= & \frac{S_{p k t}}{B\left(S_{p k t}-S_{o h}\right)\left(1-Q\left(\sqrt{2 \mathbb{K} p_{t x}}\right)\right)^{S_{p k t}}} \\
& \times\left[1-\frac{S_{p k t} \sqrt{\mathbb{K} p_{t x}} \exp \left(-\mathbb{K} p_{t x}\right)}{2 \sqrt{\pi}\left(1-Q\left(\sqrt{2 \mathbb{K} p_{t x}}\right)\right)}\right]
\end{aligned}
$$

where

$$
\mathbb{K}=\frac{\lambda^{2}}{16 \pi^{2} d^{2} p_{s}} .
$$

Let $\frac{d e\left(p_{t x}\right)}{d p_{t x}}=0$, we have

$$
2 \sqrt{\pi}\left(1-Q\left(\sqrt{2 \mathbb{K} p_{t x}}\right)\right)=S_{p k t} \sqrt{\mathbb{K} p_{t x}} \exp \left(-\mathbb{K} p_{t x}\right) .
$$

Because $B E R=Q\left(\sqrt{2 \mathbb{K} p_{t x}}\right) \ll 1$, Eq.(21) can be rewritten as

$$
2 \sqrt{\pi} \exp \left(\mathbb{K} p_{t x}\right)=S_{p k t} \sqrt{\mathbb{K} p_{t x}} .
$$

TheOREm 3. Eq.(22) has exactly one root in practice.

The proof can refer to reference [6]. Based on the analysis, we propose our algorithm for selecting transmission power for energy-oriented power control.

So, the algorithm is very easy: by solve Eq.(22), and the node set the power value to most next to the root of Eq.(22). The detail of the algorithm is omitted due to space limitation.

\subsection{Transmission power for throughput-oriented power selection}

Power Selection algorithm for Throughput-oriented power control (PST) is proposed in this subsection. To increase the throughput of a certain link, a higher transmission power level is better. But a higher power level has a counter effect to the network capacity. In this subsection, Throughput-toArea-Ratio (TAR) is used as a heuristic metric. Note that in this subsection, we suppose the node is distributed in a planar with uniform distribution. TAR is defined as follows:

$$
T A R\left(p_{t x}\right)=\frac{t\left(p_{t x}\right)}{A\left(p_{t x}\right)}
$$

where $A\left(p_{t x}\right)$ is the area as shown in Figure (1) and $t\left(p_{t x}\right)$ is defined in Eq.(15). To simplify the analysis, the "footprint" of a node is abstracted as a hexagon, just as the station in cellular communication. The superscript is omitted. As

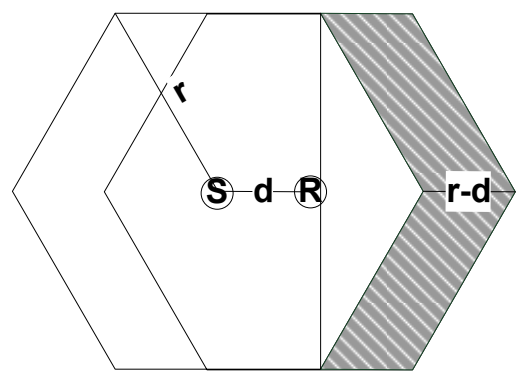

Figure 1: the "footprint" of node S and R. d is the node distance and $r$ is the carrier sensing range, respectively.

shown in Figure (1), the area covered by the two hexagons is

$$
A\left(p_{t x}\right)=\frac{3 \sqrt{3}}{2} r^{2}\left(p_{t x}\right)+\sqrt{3} r\left(p_{t x}\right)\left(r\left(p_{t x}\right)-d\right) .
$$

Put Eq.(24) and Eq.(15) into Eq.(23), we have

$$
T A R\left(p_{t x}\right)=\frac{B\left(S_{p k t}-S_{o h}\right)}{S_{p k t}} \frac{\left(1-Q\left(\sqrt{2 K p_{t x}}\right)\right)^{S_{p k t}}}{\left(\frac{5 \sqrt{3}}{2} r^{2}\left(p_{t x}\right)-\sqrt{3} d r\left(p_{t x}\right)\right)},
$$

where $\mathbb{K}$ is defined as Eq.(20) and $B$ is the bandwidth, respectively. The differential coefficient of Eq.(25) is

$$
\begin{aligned}
& \frac{d T A R\left(p_{t x}\right)}{d p_{t x}}=\frac{B\left(S_{p k t}-S_{o h}\right)}{S_{p k t}} \frac{\left(1-Q\left(\sqrt{2 K p_{t x}}\right)\right)^{S_{p k t}-1}}{\left(\frac{5 \sqrt{3}}{2} r^{2}\left(p_{t x}\right)-\sqrt{3} d r\left(p_{t x}\right)\right)} \times \\
& \left(\frac{S_{p k t} \sqrt{\mathbb{K}} \exp \left(-\mathbb{K} p_{t x}\right)}{2 \sqrt{\pi p_{t x}}}-\frac{\left(1-Q\left(\sqrt{2 \mathbb{K} p_{t x}}\right)\right)\left(5 \sqrt{3} r\left(p_{t x}\right)-\sqrt{3} d\right)}{\left(\frac{5 \sqrt{3}}{2} r^{2}\left(p_{t x}\right)-\sqrt{3} d r\left(p_{t x}\right)\right)} r^{\prime}\left(p_{t x}\right)\right)
\end{aligned}
$$

According to Eq.(1), we have

$$
p_{c s}=\frac{\lambda^{2}}{16 \pi^{2} r^{2}} p_{t x}
$$

where $p_{c s}$ is the carrier sensing threshold. Hence,

$$
\begin{gathered}
r\left(p_{t x}\right)=\frac{\lambda}{4 \pi} \sqrt{\frac{p_{t x}}{p_{c s}}}, \\
\frac{d r\left(p_{t x}\right)}{p_{t x}}=\frac{\lambda}{8 \pi} \sqrt{\frac{1}{p_{t x} p_{c s}}} .
\end{gathered}
$$

Let $\frac{d T A R\left(p_{t x}\right)}{d p_{t x}}=0$, we have

$$
\sqrt{\pi} \exp \left(\mathbb{K} p_{t x}\right)\left(1+\frac{5}{5-2 \sqrt{\frac{p_{c s t h}}{\mathbb{K} p_{t x} p_{s}}}}\right)=S_{p k t} \sqrt{\mathbb{K} p_{t x}},
$$

where $p_{\text {csth }}$ is the carrier sensing threshold. Eq.(30) is similar to Eq.(22). And we have the following theorem.

Theorem 4. Eq.(30) has exactly one root in practice.

PST is very similar to PSE except solving two different equations, so the detail of PST is omitted too.

\subsection{Some implementation issues}

In this subsection, we consider some implementation issues for PSE and PST.

1. How to get the parameters in Eq.(20).

In ad hoc networks, every node broadcasts periodically to help the node in the network find a route. Hence, 
$1 \longrightarrow 2 \quad 3 \longrightarrow 4 \quad \cdots \cdots \quad 27 \longrightarrow 28 \quad 29 \longrightarrow 30$

Figure 2: Chain topology : 30 nodes with 15 flows. The arrow between two nodes indicates traffic flows.

the node coordinate used to compute $d$ can be added into the broadcast message. As for $p_{s}$ in 802.11, the network card will sample channel power to detect if the channel is idle or not. Based on the sampled power, the node can estimate noise and interference power using the scheme proposed in [7]. Different from the scheme in [7] which is used to estimate the signal power that is higher than carrier sensing threshold, we use it to estimate the signal power that is lower than carrier sensing threshold. The estimated power is added in the control packets, namely, RTS, CTS and ACK packets.

2. The computation of minimum transmission power in the algorithms.

In PSE, Eq.(22) is only relevant to packet size. To accelerate the running speed and reduce energy consumption, $\mathbb{K} p_{t x}$ can be resolved according to the packet size in advance, and these values are written in ROM. A value is 8 bytes and there are less than 1500 values which is the value of the maximum payload size of 802.11 , so the ROM size is $12 \mathrm{~KB}$. In practical implementation, $p_{t x}$ can be computed according to $\mathbb{K} p_{t x}$ from ROM and $\mathbb{K}$. For PST, the energy consumption is not the most important. $\mathbb{K} p_{t x}$ is resolved by numeric algorithms. In our simulation, $\mathbb{K} p_{t x}$ in Eq.(30) can be resolved with iterating no more than 20 times.

\section{PERFORMANCE EVALUATION}

In this section, we evaluate our algorithms and compare them with 802.11 standard MAC and the following two abstract power control schemes:

- Power control with minimum transmission power, denoted as PC min-Tx, for example [8] [9] [10].

- Power control with certain SINR. In this power control, the SINR is kept in a value to keep the performance, for example [11]. In this paper, we simulate the scheme with $\mathrm{SINR}=4.5,10,20$, denoted as $\mathrm{PC} \operatorname{SINR}=4.5, \mathrm{PC}$ $\mathrm{SINR}=10$ and $\mathrm{PC}$ SINR $=20$ respectively.

\subsection{Evaluation metrics}

Because the purposes of power control are to improve the energy efficiency and network throughput, we use the following two metrics to evaluate the algorithms referred above:

Data transmitted per Joule: The valid data delivered successfully per unit of transmission energy consumption. This metric is used to evaluate the energy efficiency. It is calculated as the total data delivered divided by the total amount of transmission energy consumption over all the nodes. In this paper, the energy consumed in packet reception and processing is not counted in the above metric. In this metric, valid data delivered successfully does not include the packet discarded and the packet overhead.

Aggregate throughput: The valid data delivered successfully by the network. This metric is used to evaluate the bandwidth efficiency. It is calculated as the total data delivered by the network over the simulation time.

\subsection{Simulation Model}

To better simulate the algorithms, we develop a simulator written by $\mathrm{C}++$. In our simulator, the impact of noise and bit error are taken into account. The transmission range is $250 \mathrm{~m}$, carrier sensing range is $550 \mathrm{~m}$, maximum transmission power is $0.2818 \mathrm{~W}$, receiving threshold power is $4.46 \mathrm{e}-10 \mathrm{~W}$ and carrier sensing power is $9.21 \mathrm{e}-11 \mathrm{~W}$. Other the parameters are referred from 802.11 specification [4]. For every set of data, i.e., the energy efficiency and throughput for every packet size on a specific topology, we simulated 100s. In our simulator, to simplify, we use a static routing protocol because power selection algorithms run over MAC layer. Note that in this paper, we suppose that the asymmetric problem introduced by power control has been solved, so a busy tone channel with bandwidth of $64 \mathrm{kbps}$ is introduced to solve the hidden terminal problem in our simulation. The node is static, but this work can be easily extend to mobile node by multiplying a constant for every power as it was done in [9].

\subsection{Simulation topology}

In this paper, the algorithms are simulated with chain topology, grid topology and random topology, respectively.

1. Chain topology

Figure 2 shows our chain topology, which consists of 30 nodes. In Figure 2, there are 15 flows, namely, node 1 and 2 , node 3 and $4, \ldots$, node 29 and 30 , respectively. The distance between adjacent nodes is uniform. In our simulation, we set the distance $50 \mathrm{~m}, 100 \mathrm{~m}, 150 \mathrm{~m}$ and $200 \mathrm{~m}$, respectively.

2. Grid topology

We simulate the algorithms on three grid topologies with node density from dense to sparse.

Topology 1: $10^{*} 10$ nodes, the distance between adjacent nodes is $60 \mathrm{~m}$.

Topology 2: $6 * 6$ nodes, the distance between adjacent nodes is $120 \mathrm{~m}$.

Topology $3: 5 * 5$ nodes, the distance between adjacent nodes is $180 \mathrm{~m}$.

The grid topology is an extension of chain topology. And due to the space limitation, the grid topologies are not shown in this paper.

3. Random topology Figure 3 shows the random topology of our simulation. The nodes are distributed in a $1000 * 1000 \mathrm{~m}^{2}$ plane randomly with uniform distribution. In Figure 3, the two node pairs with red lines indicate that they are out the transmission range of each other. In our simulation, we suppose there is no forwarding node between any two nodes.

\subsection{Simulation results}

\subsubsection{Chain topology}

Figure 4 and 5 show the simulation results for 30 nodes with 15 flows. X-axis represents the length of packet and Y-axis represents the performance metrics (Mbits per Joule and throughput). The results show that PSE and PST performs better than traditional power selection algorithms and 802.11. As shown in Figure 4 and 5, PSE performs better 


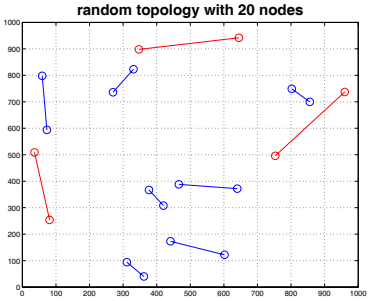

(a) 20-node topology

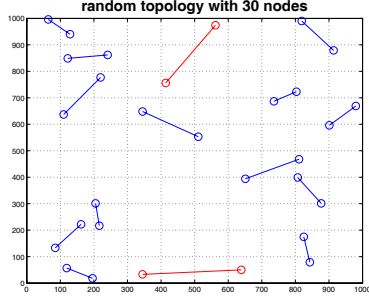

(b) 30-node topology

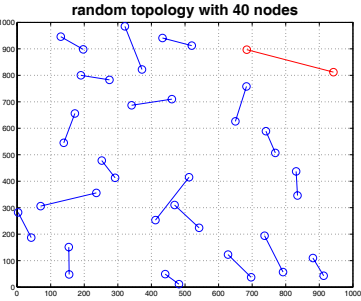

(c) 40-node topology

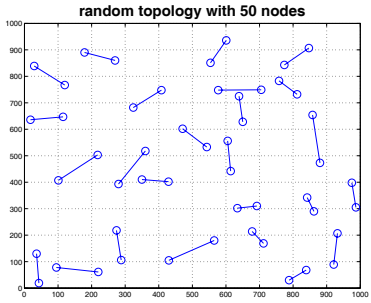

(d) 50-node topology

Figure 3: random topology. There are 20, 30, 40 and 50 nodes distributed in a $1000 * 1000 m^{2}$ plane randomly. The red line means that the two nodes are out the transmission range of each other.

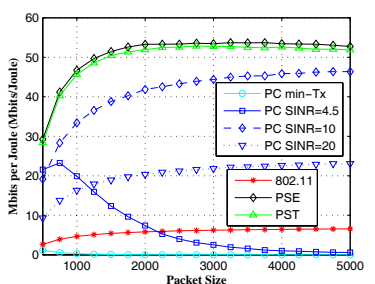

(a) distance $=50 \mathrm{~m}$

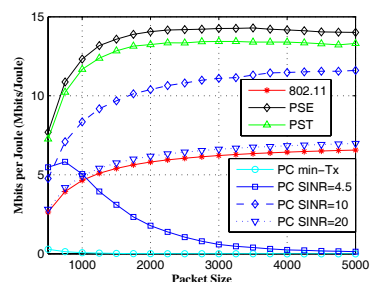

(b) distance $=100 \mathrm{~m}$

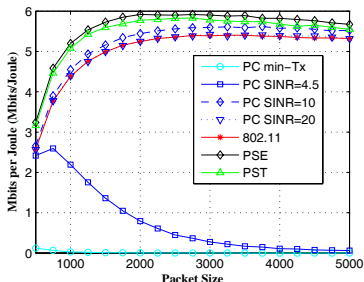

(c) distance $=150 \mathrm{~m}$

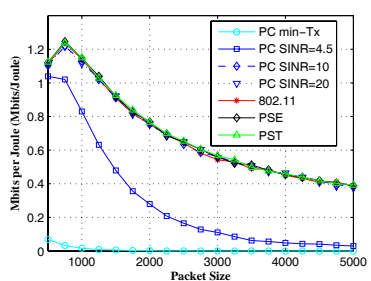

(d) distance $=200 \mathrm{~m}$

Figure 4: energy consumption for chain topology. PSE and PST perform better than other algorithms. The performance gap becomes smaller with the increasing of node distance.

than PST in terms of energy efficiency and PST performs better than PSE in terms of throughput.

Energy efficiency: Figure(4a-4d) depict the valid data received per joule energy for different packet size and node distance. For PSE and PST, a larger packet yields higher energy efficiency. The shorter the node distance is, the more evident the energy efficiency is. As shown in Figure 4d, when node distance reaches $200 \mathrm{~m}$, the performance of PSE, $\mathrm{PST}, \mathrm{PC} \mathrm{SINR}=10, \mathrm{PC} \mathrm{SINR}=20$ and 802.11 are the same because of the same transmission power. And the trace file shows that for these 5 algorithms set the transmission power the maximum value- $p_{\max }$.

Aggregate throughput: Figure(5a-5d) depict the aggregate throughput for difference packet size and node distance. As shown in the figure, the throughput of PST is higher than other algorithms. And the throughput gap is not evident for PST, PSE, PC SINR=10 and PC SINR=20, because the capacity is the same for these algorithms in uniform linear topology. In PC SINR $=10$ and PC $\mathrm{SINR}=20$, the power level is higher, so the interference in the network becomes higher than that of PST. Hence, despite the same capacity, the throughput in PC SINR $=10$ and PC SINR $=20$ is slightly lower than that in PST.

\subsubsection{Grid topology}

The simulation results for grid topologies are shown in Figure 6 and 7. As shown in the figures, PSE and PST outperforms other algorithms for diverse node distribution. For sparse distribution, most algorithms (except $\mathrm{PC} \mathrm{SINR}=4.5$ and PC Tx-min) perform the same due to the same transmission power. In Figure (6a-6c), the energy efficiency decreases with the node number decreases, because of the increase of node distance. In Figure 7, as the number of nodes decreases, the aggregate valid throughput decrease due to the decreasing of concurrent flows.

\subsubsection{Random topology}

Figure 8 and 9 show our simulation results for random topology with 20,30, 40 and 50 node, respectively. In Figure 8, PSE shows a better performance in terms of energy efficiency. And as shown in Figure 9, PST performs better than other algorithms. In Figure 8 and 9 , the denser the node distribution is, the better the algorithms performs except for 802.11 and PC min-Tx which perform the best in 30 nodes topology. This is because 802.11 and PC minTx has a larger capacity in 30-node topology than 20-node topology and much less collision and packet error compared with 40-node and 50-node topology. While for PSE and PST, by selecting appropriate power, the capacity increasing overcomes the effect of collision and packet error. As shown in Figure 8c and Figure 9c, PSE and PST has the same throughput, but PSE performs better than PST in terms of energy efficiency. This is because that PSE has a lower packet error. The trace file shows that, for 40-node topology, PST has a larger packet drop ratio.

\subsubsection{Some common observations}

We can come to some common observations for the above results we presented.

First, our proposed algorithms have better performance than traditional power control algorithms and 802.11. The algorithms are simulated with different node distance, node distribution and node density. The results shows that PSE outperforms other algorithms in terms of energy efficiency and PST has better performance than other algorithms in terms of network throughput.

Second, the efficiencies of PSE, PST, 802.11 and PC SINR increase with packet size when the node distance is short. As the node distance becomes shorter or node distribution becomes denser, all the power control algorithms perform better. When the node distance is too large, the energy efficiency decreases with the packet size, e.g., Figure $4 d$ and Figure 6c. 


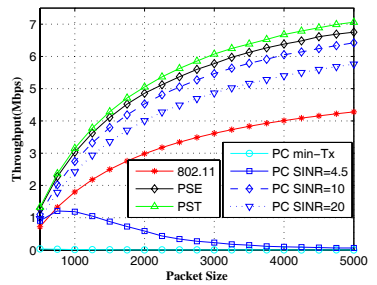

(a) distance $=50 \mathrm{~m}$

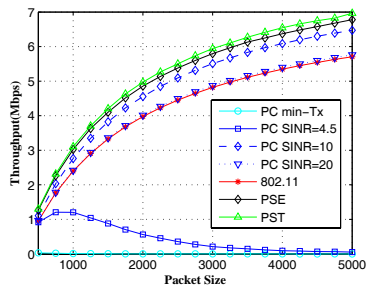

(b) distance $=100 \mathrm{~m}$

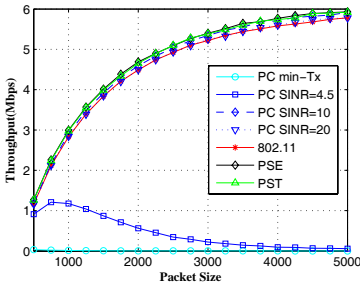

(c) distance $=150 \mathrm{~m}$

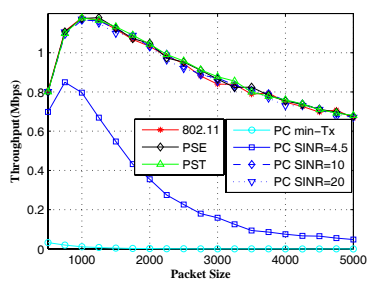

(d) distance $=200 \mathrm{~m}$

Figure 5: aggregate throughput for chain topology. PSE and PST increase the network throughput. For short distance, aggregate throughput increases with the increasing of packet size.

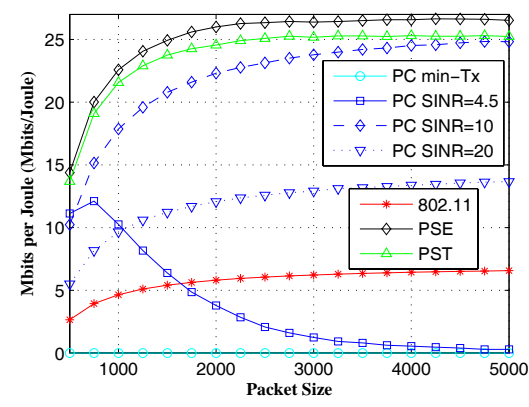

(a) $10 * 10$ nodes, $60 \mathrm{~m}$

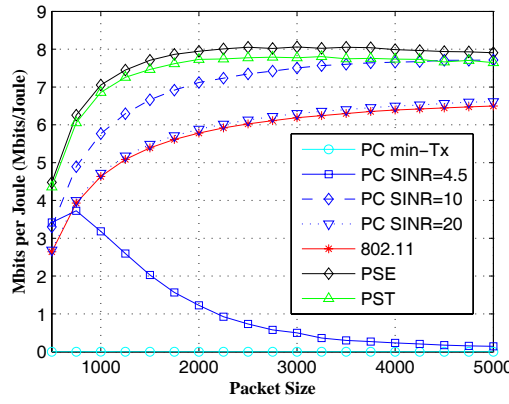

(b) $6 * 6$ nodes, $120 \mathrm{~m}$

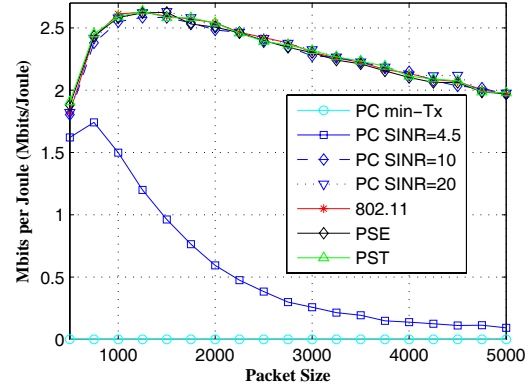

(c) $5 * 5$ nodes, $180 \mathrm{~m}$

Figure 6: energy consumption for grid topology.PSE and PST yield a better energy efficiency than other algorithms on grid topology.

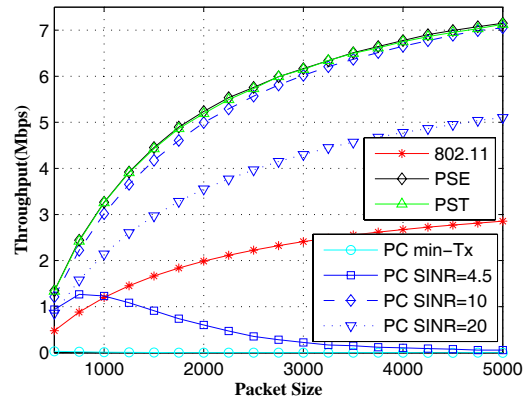

(a) $10 * 10$ nodes, $60 \mathrm{~m}$

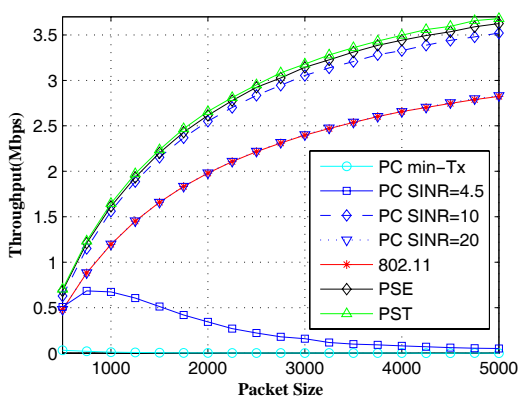

(b) $6 * 6$ nodes, $120 \mathrm{~m}$

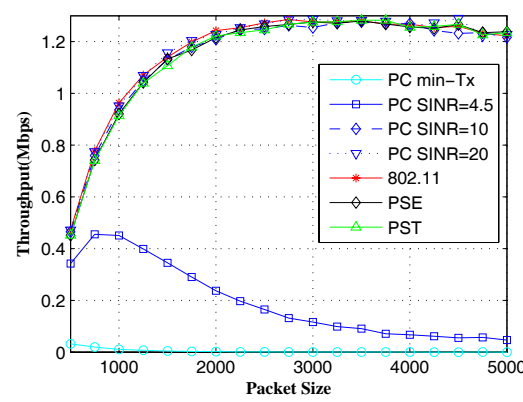

(c) $5 * 5$ nodes, $180 \mathrm{~m}$

Figure 7: aggregate throughput for grid topology. PSE and PST have a higher aggregate throughput than other algorithms on grid topology.

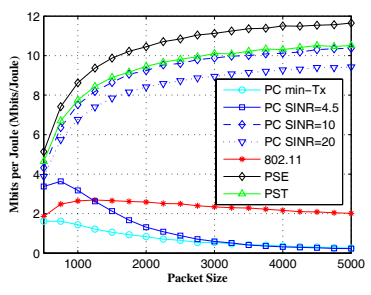

(a) 20 nodes

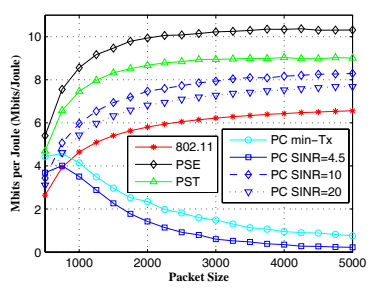

(b) 30 nodes

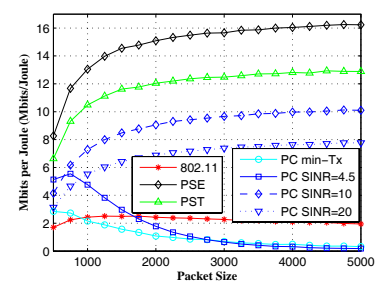

(c) 40 nodes

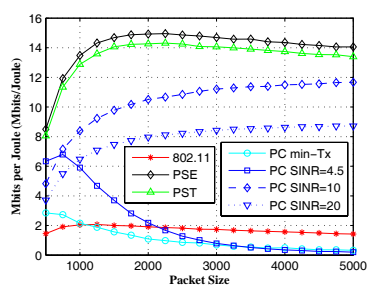

(d) 50 nodes

Figure 8: energy consumption for random topology. PSE and PST have a better energy efficiency than other algorithms on random uniform topology. 


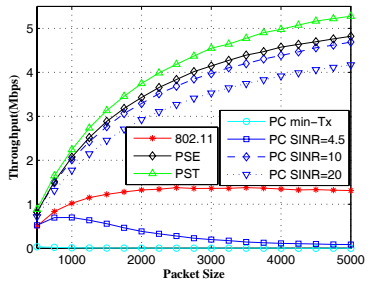

(a) 20 nodes

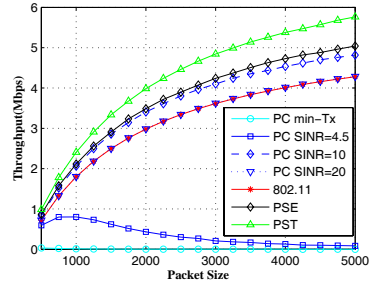

(b) 30 nodes

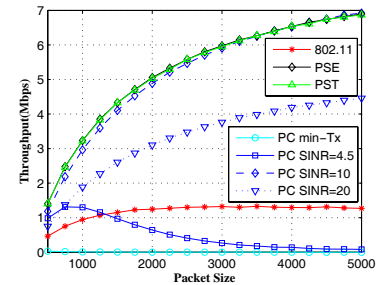

(c) 40 nodes

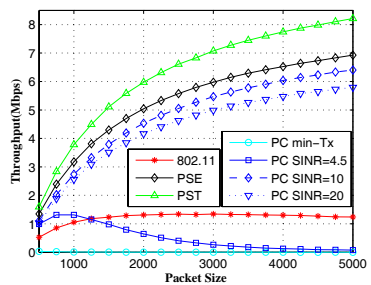

(d) 50 nodes

Figure 9: aggregate throughput for random topology. PSE and PST have a higher aggregate throughput than other algorithms on random uniform topology.

\section{RELATED WORK}

Power control is a widely used technology to improve the Quantity of Service (QoS) of the wireless networks, e.g. decreasing the energy consumption and improving network throughput. In this section, we will summarize the previous works most related to this paper.

\subsection{Power Control for energy conservation}

A number of studies have been carried out on power control for energy conservation [12] [8] [13] [9] [14] [15] [11]. A simple power control is the use of maximum power for RTSCTS handshake and minimum power required for communication for DATA and ACK [12] [8] [13]. In [12], the sender adds its current transmission power in its RTS packet. The receiver then computes the appropriate power for communication and includes this power in CTS packet. To decrease the potentially increased energy consumption, Jung and Vaidya [9] proposed that the transmission power of DATA should be increased to the maximum periodically. In the aforementioned schemes, negotiating transmission power for every transmission may consume too much energy or bandwidth. In [16], Pires et al. proposed an algorithm that attaches a table to each node, which stores the transmission power used on previous transmissions. The above studies assume the channel and transceiver parameters (e.g., noise, interference and battery energy) are invariant. However, in practice, the battery power decreases with time and the channel varies with time and environment. To prolong the network's life and minimize end-to-end delay, Meghanathan and Farago [14] proposed a battery power awareness power control, each node starts with a higher transmission power and tunes down this power gradually depending on the battery power available. Recently, some researches [17] [11] [15] propose that the transmission power should change with the time and environment. For example, the power attenuation is different for indoor and outdoor environments; the noise and interference vary with the network throughput. Considering the variability of channel, Lin [17] proposed ATPC. ATPC uses a pairwise transmission power control algorithm. In [11], transmission power is determined by node interaction in some constraints (e.g., the maximum transmission power, the minimum receiving power at the receiver, the minimum receiving SINR at the receiver). In [15], an adaptive power control is proposed. Initially, the data is transmitted at the maximum power level, if this data is received successfully, then transmission power decreases a constant value; if transmitting time expires at the frame sender, its power level increases a constant value. In [18], the authors developed distributed algorithms which jointly allocate frequency spectrum, transmission powers, traffic input rates, and traffic routes on a node-by-node basis to minimize total cost in an interference-limited multi-hop wireless network. Recently, there are power control algorithms proposed for directional antenna [19] [20].

\subsection{Power control for throughput increment}

Using power control, the interference range of the node will reduce. This may increase the network capacity. Gupta and Kumar [5] pointed out that, by tuning the transmission power, power control improves aggregate channel utilization may be improved up to a factor of $O(\rho)$, where $\rho$ is the node density in the region. Monks et al. proposed PCMA protocol [21]. PCMA generalized the transmit-or-defer "on/off" collision avoidance model of 802.11 to a more flexible "variable bounded power" collision suppression model. In PCMA, the transmitter- receiver pairs adjust the power to the minimum required for a successful transmission. Muqattash et al. proposed PCDC [22]. By overhearing RTS and CTS packets, PCDC protocol estimates the future interference transmissions, and adjusts the transmission power according to these potential transmissions. In POWMAC [23], every node treats the aggregate interference and noise power to the thermal noise as the network load factor. The transmission power is computed according to the receiver's network load factor. Kim et al's [22] studied the power control for multi-rate networks. Because transmission can affect the spatial reuse and data rate, the transmission power is set to the optimal value that balances the two factors. In [10], using software defined radio (SFD), the channel is divided into several sub-channel. Shi and Hou [10] proposed channel scheduling algorithm for power control. The transmission power is set to the minimum power for communication.

Recently, there are some works focusing on selecting the transmission power [24] [25]. In [24], Dai proposed 3 algorithms to find the minimum uniform transmission power of an ad hoc wireless network under the assumption that each node uses the same transmission power, while maintaining network connectivity. Similar to the assumption in [24], Chen [25] aims to minimize the end-to-end power consumption.

\section{CONCLUSION}

In this paper, we focus on selecting an appropriate transmission power for wireless ad hoc networks. We model the network under the assumption that the channel is AWGN and transceivers use BPSK modulation. In the models, we take into account the network noise and interference, packet size and node distribution. The models show that obtaining the optimal transmission power is equivalent to a nonlinear integer programming problem which is NP-hard. Then we propose two heuristic power selection algorithms, PSE for energy-oriented power control and PST for throughput- 
oriented power control. The two algorithms are $O(1)$ time complexity and local. In PSE, the sender minimizes the transmission energy consumed for transmitting a valid bit successfully. In PST, the sender maximizes the valid bits transmitted successfully per unit area. Then we prove that there is exactly one transmission power for PSE and PST, respectively. Based on the analysis, two transmission power selection algorithms are proposed in this paper. At last, we compare our algorithms with traditional power control ones. Simulation results show that PSE and PST achieve energy saving and throughput increasing compared with traditional power control and 802.11.

Although PSE and PST improve the performance of power control, it does not get the optimal efficiency. The future work includes developing more efficient power selection algorithms. As discussed in this paper, our algorithms are local and based on the assumption that all the node are cooperative. The future work will take selfish node and fairness into consideration, too.

\section{ACKNOWLEDGEMENT}

This work is supported in part by Supported by the National High-Tech Research and Development Plan of China(863) under Grant No.2006AA01Z209 and No.2007AA01Z2A2 and the National Grand Fundamental Research Program of China (973) under Grant No.2003CB314807. We also thanks to the anonymous reviewers for their constructive feedback.

\section{REFERENCES}

[1] S. Choudhury and J. D. Gibson, "Payload length and rate adaptation for multimedia communications in wireless lans," IEEE JOURNAL ON SELECTED AREAS IN COMMUNICATIONS (J-SAC), vol. 25, no. 4, pp. 796-807, 2007.

[2] A. Goldsmith, "Wireless commonication," pp. 40-41, 2005.

[3] ——, "Wireless commonication," pp. 160-161, 2005.

[4] I. C. Society, "Wireless lan medium access control (mac) and physical layer (phy) specications," in IEEE standard 802.11, 1999.

[5] P. Gupta and P. R. Kumar, "The capacity of wireless networks," in IEEE Transactions on Information Theory, March 2000, pp. 46(2):388-404.

[6] J. He, J. Yang, and C. An, "Transmission power selection for ad hoc networks," in Technical Report, http://nmgroup.tsinghua.edu.cn/ jpHe/tr-powerselection.pdf, 2008.

[7] S. C. Javier del Prado Pavon, "Link adaptation strategy for ieee 802.11 wlan via received signal strength measurement," in Proc. of IEEE ICC, Seattle, Washington, USA, May 2003, pp. 1108-1113.

[8] S. Agarwal, S. Krishnamurthy, R. H. Katz, and S. K. Dao, "Distributed power control in ad-hoc wireless networks," in Proc. of IEEE PIMRC, San Diego, California, USA, Sept. 2001, pp. F-59 - F-66.

[9] E.-S. Jung and N. H. Vaidya, "A power control mac protocol for ad hoc networks," in Proc. of ACM MobiCom, Georgia, USA, Sept. 2002, pp. 36-47.

[10] Y. Shi and Y. T. Hou, "Optimal power control for multi-hop software defined radio networks," in Proc. of IEEE Infocom, Anchorage, Alaska, USA, May 2007, pp. 1694-1702.

[11] L. H. A. Correia, D. F. Macedo, D. A. C. Silva, A. L. dos Santos, and A. A. F. L. et al, "Transmission power control in mac protocols for wireless sensor networks," in Proc. of ACM MSWiM, Montreal,Quebec, Canada, Oct. 2005, pp. 282-289.

[12] M. B. Pursley, H. B. Russell, and J. S. Wysocarski, "Energy-efficient transmission and routing protocols for wireless multiple-hop networks and spread-spectrum radios," in Proc. of EUROCOMM, 2000, pp. $1-5$.

[13] J. Gomez, A. T. Campbell, M. Naghshineh, and C. Bisdikian, "Conserving transmission power in wireless ad hoc networks," in Proc. of IEEE ICNP, Washington DC,USA, November 2001, pp. 24-32.

[14] N. Meghanathan and A. Farago, "Power sensitive power control in ad hoc networks," in Proc. of ACM Southeast Conference, Kennesaw, Georgia, USA, March 2005, pp. 81-86.

[15] E. Lopez-Aguilera and J. Casademont, "Transmit power control mechanisms in ieee 802.11cellular networks," in Proc. of ACM IWCMC, Vancouver, British Columbia, Canada, July 2006, pp. 731-736.

[16] A. A. Pires, J. F. de Rezende, , and C. Cordeiro, "Alca: A new scheme for power control on 802.11 ad hoc networks," in Proc. of IEEE WoWMoM, Taormina Giardini, Naxos, June 2005, pp. 457-477.

[17] S. Lin, J. Zhang, G. Zhou, L. Gu, T. He, and J. A. Stankovic, "Atpc: Adaptive transmission power control for wireless sensor networks," in Proc. of ACM Sensys, Boulder, Colorado, USA, November 2006, pp. 223-236.

[18] Y. Xi and E. M. Yeh, "Distributed algorithms for spectrum allocation, power control,routing, and congestion control in wireless networks," in Proc. of ACM MobiHoc, Montreal, Quebec, Canada, Sept. 2007, pp. 180-189.

[19] Z. Huang, Z. Zhang, and B. Ryu, "Power control for directional antenna-based mobile ad hoc networks," in Proc. of IEEE IWCMC, Vancouver, British Columbia, Canada, July 2006, pp. 917-922.

[20] Z. Zhang, "Pure directional transmission and reception algorithms in wireless ad hoc networks with directional antennas," in Proc. of IEEE ICC, Seoul, Korea, May 2005, pp. 3386-3390.

[21] J. P. Monks, V. Bharghavan, and W. mei W. Hwu, "A power controlled multiple access protocol for wireless packet networks," in Proc. of IEEE Infocom, Anchorage, Alaska, USA, April 2001, pp. 219-228.

[22] Y.-S. Kim, H. Lim, and J. C. Hou, "Improving spatial reuse throughput tuning transmit power, carrier sense threshold, and data rate in multihop wireless networks," in Proc. of ACM MobiCom, Los Angeles, California, USA, Sept. 2006, pp. 366-377.

[23] A. Muqattash and M. Krunz, "Single-channel solution for transmission power control in wireless ad hoc networks," in Proc. of ACM MobiHoc, Roppongi Hills, Tokyo, Japan, May 2004, pp. 210-221.

[24] Q. Dai and J. Wu, "Computation of minimal uniform transmission power in ad hoc wireless networks," in Proc of ICDCSW'03, Providence, Rhode Island, USA, May 2003, pp. 680-684.

[25] Y. Chen, E. G. Sirer, and S. B. Wicker, "On selection of optimal transmission power for ad hoc networks," in Proc. of HICSS'03, Big Island, Hawaii, USA, Jan. 2003, pp. 10-19. 